\title{
Fast high-resolution terahertz radar imaging at 25 meters
}

Ken B. Cooper, Robert J. Dengler, Nuria Llombart, Ashit Talukder, Anand V. Panangadan, et al.

Ken B. Cooper, Robert J. Dengler, Nuria Llombart, Ashit Talukder, Anand V. Panangadan, Chris S. Peay, Imran Mehdi, Peter H. Siegel, "Fast highresolution terahertz radar imaging at 25 meters," Proc. SPIE 7671, Terahertz Physics, Devices, and Systems IV: Advanced Applications in Industry and Defense, 76710Y (26 April 2010); doi: 10.1117/12.850395

EDIE Event: SPIE Defense, Security, and Sensing, 2010, Orlando, Florida, United States 


\title{
Fast, high-resolution terahertz radar imaging at 25 meters
}

\author{
Ken B. Cooper*a ${ }^{*}$, Robert J. Dengler ${ }^{\mathrm{a}}$, Nuria Llombart ${ }^{\mathrm{b}}$, Ashit Talukder ${ }^{\mathrm{a}}$, Anand V. Panangadan ${ }^{\mathrm{a}}$, \\ Chris S. Peay ${ }^{\mathrm{a}}$, Imran Mehdi ${ }^{\mathrm{a}}$, Peter H. Siegel ${ }^{\mathrm{a}}$ \\ ${ }^{a}$ Jet Propulsion Laboratory, California Institute of Technology, 4800 Oak Grove Dr., Pasadena, CA, \\ USA 91109-8099; \\ ${ }^{\mathrm{b}}$ Universidad Complutense de Madrid, Av. Arcos de Jalón 118, 28037 Madrid
}

\begin{abstract}
We report improvements in the scanning speed and standoff range of an ultra-wide bandwidth terahertz (THz) imaging radar for person-borne concealed object detection. Fast beam scanning of the single-transceiver radar is accomplished by rapidly deflecting a flat, light-weight subreflector in a confocal Gregorian optical geometry. With RF back-end improvements also implemented, the radar imaging rate has increased by a factor of about 30 compared to that achieved previously in a $4 \mathrm{~m}$ standoff prototype instrument. In addition, a new $100 \mathrm{~cm}$ diameter ellipsoidal aluminum reflector yields beam spot diameters of approximately $1 \mathrm{~cm}$ over a $50 \times 50 \mathrm{~cm}$ field of view at a range of $25 \mathrm{~m}$, although some aberrations are observed that probably arise from misaligned optics. Through-clothes images of concealed pipes at $25 \mathrm{~m}$ range, acquired in 5 seconds, are presented, and the impact of reduced signal-to-noise from an even faster frame rate is analyzed. These results inform the requirements for eventually achieving sub-second or video-rate $\mathrm{THz}$ radar imaging.
\end{abstract}

*ken.b.cooper@jpl.nasa.gov; phone 1818 354-4371; fax 1818 393-4683

Keywords: terahertz imaging radar, standoff concealed weapons detection

\section{INTRODUCTION}

The investigation of $\mathrm{THz}$ or submillimeter-wave radiation for person-borne concealed weapons or explosives detection has burgeoned over the last decade as microwave source and detector technology reach ever higher frequencies ${ }^{1}$. Three core paradigms of submillimeter-wave sensing are spectroscopic signatures, passive imaging, and active imaging. Wideband $\mathrm{THz}$ spectroscopy has shown some promise for eventual standoff detection of explosives ${ }^{2}$, but demonstration of remote THz spectroscopic sensing has not been made yet. Passive thermal sensing in the $100-300 \mathrm{GHz}$ range is the most mature technology, with several companies now offering near-video rate imaging systems ${ }^{3}$ and with substantial non-commercial development work as well ${ }^{4}$. However, drawbacks of the passive detection approach include an unfavorable tradeoff between threat contrast and image resolution at frequencies exceeding $100 \mathrm{GHz}$, as well as weak signals from objects concealed by multiple layers of clothing.

These drawbacks can be partially overcome, in principle, by using active imagers. In an active imager, strong scene illumination can overcome the low contrast of passive detection, especially at high frequencies where spatial resolution is the greatest. Penetration through thick clothing can be also accomplished by increasing the transmitting power. Unlike passive detectors, active transceivers will confront no fundamental detection sensitivity limit determined by a scene's thermal background temperature; a very high signal-to-noise ratio (SNR) can be achieved given a strong enough illumination source. However, compact solid-state sources can typically only generate about $\sim 1 \mathrm{~mW}$ up to $\sim 1 \mathrm{THz}^{5}$ giving an upper limit to the radiation's penetrating ability. More importantly, active THz through-clothes imagers, particularly those that rely on coherent illumination and detection, must overcome large signal clutter and speckle caused by a scene with a diversity of angles of incidence, surface roughness, and layers of concealing clothing.

The active THz radar approach being developed at the Jet Propulsion Laboratory (JPL) ${ }^{6}$ and elsewhere ${ }^{7,8}$ addresses the clutter and speckle problem by probing the three-dimensional cross-sectional range profile of a target, rather than just measuring reflectivity contrast in two dimensions, as a conventional active imager might. JPL's first prototype THz imaging radars operated at 0.60 and $0.67 \mathrm{THz}$, and they used a $40 \mathrm{~cm}$ diameter main aperture to focus the radar beam onto a target at a $4 \mathrm{~m}$ standoff range. At this range, the beam's half-power diameter was about $0.5 \mathrm{~cm}$. Images were

Terahertz Physics, Devices, and Systems IV: Advanced Applications in Industry and Defense, edited by Mehdi Anwar, Nibir K. Dhar, Thomas W. Crowe, Proc. of SPIE Vol. 7671, 76710Y. (C) 2010 SPIE · CCC code: 0277-786X/10/\$18 - doi: 10.1117/12.850395 
generated by scanning the beam in a serpentine pattern over a scene using two large-capacity motors to turn the entire radar platform, including the main reflector and a large portion of the RF electronics, about the azimuth and elevation axes. Typical threat-detection imagery required 150-300 seconds to acquire using this system.

In this paper, we describe the implementation of a second-generation $0.67 \mathrm{THz}$ imaging radar capable of imaging a person's torso $(50 \times 50 \mathrm{~cm})$ in 5 seconds, with $1 \mathrm{~cm}$-scale resolution, at a standoff range of $25 \mathrm{~m}$. This represents an improvement in imaging speed and standoff range by factors of about 30 and 6 , respectively. Improving these performance metrics required re-designing the radar's scanning optics, increasing its main aperture diameter, speeding up its chirp generation hardware, and adding an additional intermediate frequency stage to the signal detection. Based on the results of this effort, we believe that frame rates of $1 \mathrm{~Hz}$ or higher for the $\mathrm{THz}$ imaging radar will soon be reached using essentially the same architecture described here.

\section{SYSTEM DESCRIPTION AND BEAM SCANNING AT 25 M}

\subsection{Electronic and Optical Architecture}

A photograph of the $25 \mathrm{~m}$ standoff, $0.67 \mathrm{THz}$ imaging radar is shown in Fig. 1a, along with a simplified electronics block diagram in Fig. $1 \mathrm{~b}$ and a sketch of the optical geometry for fast beam scanning in Fig. 1c. The radar's back-end electronics generates a fast 36.8-38.2 GHz chirp signal which is then tripled, amplified, sextupled, and transmitted at $662-688 \mathrm{GHz}$ by the radar's $\mathrm{THz}$ transceiver. The $26 \mathrm{GHz}$ radar bandwidth ensures sub-cm range resolution, and the frequency band was chosen for good image resolution and clothing penetration in a low atmospheric loss window. Transmit and receive duplexing is accomplished with a silicon wafer etalon. The subharmonic receive mixer is pumped by a chirped local oscillator (LO) to produce an intermediate frequency (IF) signal at a frequency of $3.6 \mathrm{GHz}$ plus a shift proportional to the target range. The front-end radar waveguide blocks are a combination of JPL-built parts (110-115 GHz amplifiers and a 330-345 GHz tripler) and commercial Virginia Diodes Inc. components (110-115 GHz triplers, a 660-690 GHz sextupler chain, and a 660-690 GHz subharmonic mixer). After downconversion, the in-phase and quadrature signals are digitized in a $2.8 \mathrm{MHz}$ bandwidth and processed using standard frequency-modulated continuouswave (FMCW) radar techniques.
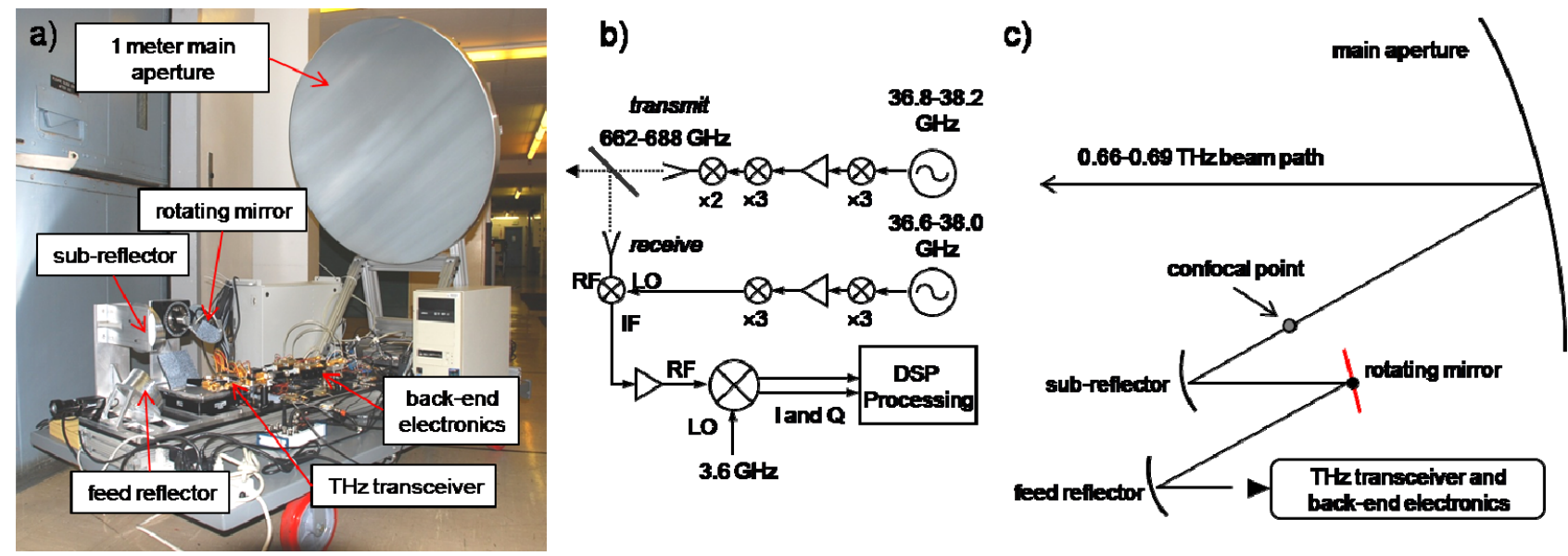

Figure 1: a) Photograph of JPL's fast-scanning, long-range $0.67 \mathrm{THz}$ imaging radar, including a $1 \mathrm{~m}$ diameter ellipsoidal main reflector and a fast-rotating mirror for beam steering. b) Simplified block diagram of the radar's $\mathrm{THz}$ transceiver and electronics. c) Optical geometry for low-distortion scanning.

The imaging radar currently utilizes only a single transceiver because $\mathrm{THz}$ heterodyne array technology for a focal plane array has not been developed. Therefore, fast imaging requires fast beam scanning and fast signal processing. An analysis of the confocal Gregorian optical design shown in Fig. 1c is described in detail elsewhere ${ }^{9}$. Briefly, the diverging $\mathrm{THz}$ beam emitted by the transceiver feed horn is focused into a parallel ray envelope using a parabolic feed reflector. These rays are then steered by a lightweight, flat two-axis rotating mirror over typically $\pm 3^{\circ}$ in elevation and 
azimuth, before proceeding to a parabolic sub-reflector and then the main aperture. The overall optical magnification reduces this deflection angle for the projected $25 \mathrm{~m}$ beam to approximately $\pm 0.5^{\circ}$. The subreflector shares its focus (confocal point in Fig. 1c) with the near-side focal point of the $1 \mathrm{~m}$ diameter ellipsoidal main aperture, whose second focal point is $25 \mathrm{~m}$ down range. The feed and sub-reflectors were diamond-turned by Corning Inc., and the $100 \mathrm{~cm}$ main reflector was machined by Custom Microwave Inc.

Although the three additional mirrors between the $\mathrm{THz}$ feed and the main aperture adds to the system complexity, there is a significant benefit for fast beam scanning. First, the 5 in. flat steering mirror has a small inertia that permits rapid nodding motion. Second, both the main aperture and all the radar electronics components remain stationary during scanning, simplifying the instrument's platform design and avoiding any spurious phase shifts from flexing coaxial cable or waveguide. This is in contrast to our first-generation $\mathrm{THz}$ imaging radar, where the front-end electronics and the main aperture were mounted separately from the back-end components on a large rotating platform. And finally, by steering the beam as an envelope of parallel rays, rather than when it is diverging toward the main reflector, optical aberrations are negligible even over the large scan range of typically 40-50 down-range beam widths.

\subsection{Beam Scanning Results}

As an initial assessment of the THz radar's scanning ability at $25 \mathrm{~m}$ standoff range, beam shape measurements were made following the initial installation of the $1 \mathrm{~m}$ main aperture. The reflector was hand-positioned using only a ruler and protractor, and so far no re-alignment of the optics has been done to optimize the beam shape. Even so, the initial beam shape measurements are very promising, as summarized in Fig. 2. Figure $2 \mathrm{a}$ shows a photograph of a beam shape calibration target consisting of a $5 \times 5$ grid of $3 \mathrm{~mm}$ diameter gold beads suspended in a wooden frame using 4 mil (102 $\mu \mathrm{m})$ diameter nylon thread. The bead spacing is $12.5 \mathrm{~cm}$ so that the entire grid covers a $50 \times 50 \mathrm{~cm}$ square.

Fig. $2 \mathrm{~b}$ shows the reflected radar power of the grid, integrated over a range swath of $4 \mathrm{~cm}$ around the beads and spanning approximately $60 \times 60 \mathrm{~cm}$ in the $x y$ plane. The color scale for Fig. 2b-e spans $50 \mathrm{~dB}$ of dynamic range and is normalized to the maximum power pixel of each image. Each image pixel was acquired using a $0.44 \mathrm{~ms}$ FMCW radar chirp. The total image scan time for the $161 \times 153$ pixel image of Fig. $2 \mathrm{~b}$ was 32 seconds, or an average of $1.3 \mathrm{~ms}$ per pixel. This is greater than the $0.44 \mathrm{~ms}$ chirp time because of the overhead of the scanning mirror's acceleration. All 25 beads are easily discernable in the radar image, with SNR typically exceeding $30 \mathrm{~dB}$. Interestingly, while the nylon suspension threads are barely visible in Fig. 2b, these threads have the potential to completely dominate the image, with SNRs exceeding 50 $\mathrm{dB}$, if they are aligned to be nearly perpendicular to the beam direction. This large return signal power from wispy nylon thread may be caused by Mie-type resonant scattering, since the thread circumference of $320 \mu \mathrm{m}$ is comparable to the beam wavelengths of $436-453 \mu \mathrm{m}$. This observation, as well as the image of Fig. $2 \mathrm{~b}$, is indicative of the high sensitivity of the heterodyne $\mathrm{THz}$ detection technique.

To the extent that the $3 \mathrm{~mm}$ diameter beads can be treated as point targets compared to the $>1 \mathrm{~cm}$ physical beam size at $25 \mathrm{~m}$, the bead shapes of Fig. $2 \mathrm{~b}$ represent the two-way radar beam pattern. The term "two-way" refers to the square of the conventional antenna intensity, which is appropriate for a radar that transmits and receives using a single antenna with identical Tx and Rx beams. The center bead of Fig. 2b, highlighted by the dashed black circle, indicates that the beam pattern is fairly circular for the nominally undeflected beam. As the beam is displaced up to $25 \mathrm{~cm}$ in the $x$ and $y$ directions, the beam shapes appear to gradually become elliptical. Also, there is a vertical asymmetry in the degree of distortion away from the undisplaced beam. This probably means that the system optics are misaligned, or, less likely, that the aluminum reflectors have shape errors. An attempt will be made to better align the system optics in the future. 
a)

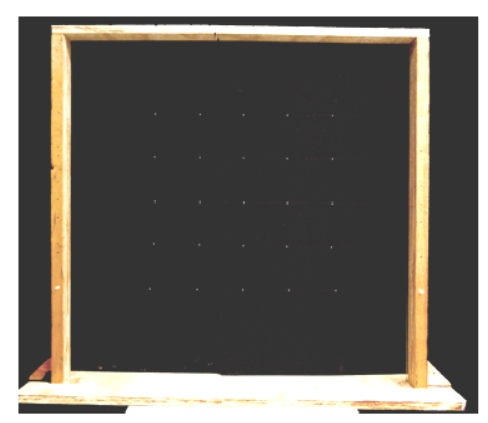

b)

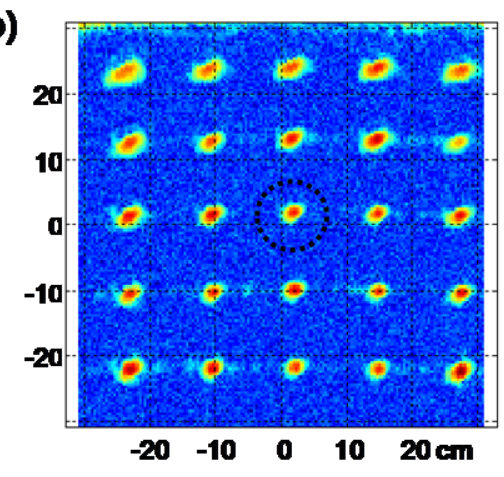

c)

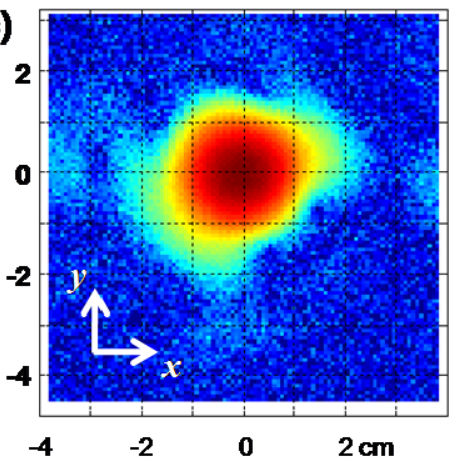

d)

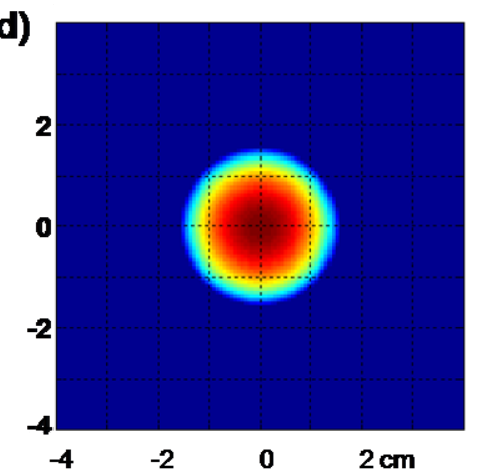

e)
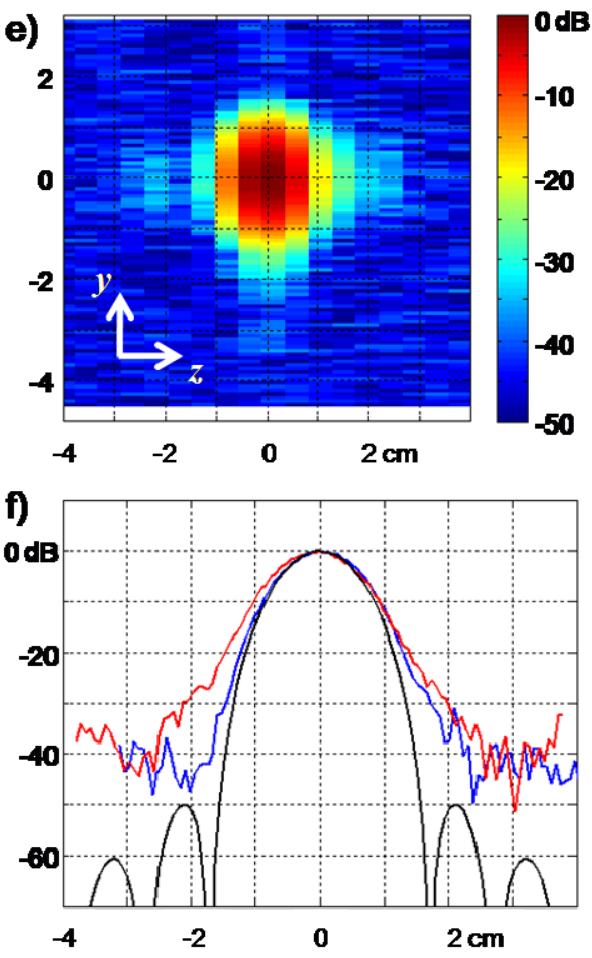

Figure 2: Initial beam shape assessment for the $25 \mathrm{~m} \mathrm{THz}$ imaging radar. a) Beam shape calibration target, a $5 \times 5$ grid of $3 \mathrm{~mm}$ diameter gold beads. b) Reflected radar power image of the beads. The outlined bead is analyzed in (c) and (e). c) Fine scan of the center bead power in the $x y$ plane, showing a nearly circular beam pattern with a $1 \mathrm{~cm}$ width at the $3 \mathrm{~dB}$ point. $\mathrm{d}$ ) Physical optics simulation of the central beam pattern, assuming a point target and identical Tx/Rx beam shapes. e) The measured bead power in the $y z$ plane, demonstrating sub-cm range resolution. f) Vertical (blue) and horizontal (red) cross-sections of the experimental data in (c), and vertical (black) cross-section of the simulated beam response from $(\mathrm{d})$.

A close analysis of the center bead pattern suggests that the overall optical system is nonetheless quite close to its design. A higher pixel-density reflected power image of this bead, integrating over a $1 \mathrm{~cm}$ swath in the $z$ direction, is shown in Fig. 2c. The image reveals a fairly circular shape that compares favorably to the two-way beam pattern of the physical optics simulation, shown below it in Fig. 2d. Vertical and horizontal cross-sections of Fig. 2c are shown in Fig. 2f, along with a vertical cross-section of the simulated beam pattern of Fig. $2 \mathrm{~d}$. Fig. $2 \mathrm{f}$ indicates that the $-3 \mathrm{~dB}$ two-way beam width is between 1.0 and $1.1 \mathrm{~cm}$, about $10 \%$ larger than the simulated diameter of $0.95 \mathrm{~cm}$. The experimental beam shape also exhibits a shallower roll-off at larger distances from the beam center. Further measurement and analysis will be required to quantify how much these discrepancies are caused by the finite width of the bead target versus misaligned optics. At displacements in $x$ and $y$ of $25 \mathrm{~cm}$, the beam shape becomes elliptical with an increase in the major axis width of typically $30-40 \%$. This is significantly larger than the physical optics simulations showing only a $1 \%$ beam width increase at these deflections ${ }^{9}$, indicating the need for further optical alignment.

Finally, Fig. 2e shows the center bead radar response in the $y z$ plane, i.e. where the horizontal distance in the image comes from the FMCW radar range power spectrum mapping detected frequency to time-of-flight, and thus to distance. The coarser pixel spacing visible in the $z$ direction of Fig. 2e is a consequence of the radar's range-compressed power spectrum being only 512 points in length, with each spectral bin representing $0.46 \mathrm{~cm}$. This is close to the theoretical range resolution (or peak width) $\Delta z$ of $0.58 \mathrm{~cm}$, from the standard FMCW radar analysis for a $26 \mathrm{GHz}$ bandwidth chirp signal: $\Delta z=c / 2 \Delta F$, where $c$ is the speed of light and $\Delta F$ is the bandwidth. The $-3 \mathrm{~dB}$ peak width of Fig. 2e is somewhat less than a single range bin. Taken together, Figs. $2 \mathrm{c}$ and $2 \mathrm{e}$ represent the three-dimensional resolution, or single-voxel volume, of the $\mathrm{THz}$ imaging radar, at the nominal zero-beam-displacement location. 
The range resolution is thus comparable to the cross-range resolution. This is a reasonable engineering goal for $\mathrm{THz}$ imaging radars because for through-clothes imagery of concealed weapons, improving the detection resolution along range yields a diminishing return of image quality if the cross-range resolution does not likewise improve, and vice versa. A convenient rule of thumb for estimating the relative values of the two resolutions is that they will be approximately equal when the ratio of the radar aperture diameter to the standoff range is equal to the ratio of the radar bandwidth to the carrier frequency. This follows from the relationship $\Delta z=c / 2 \Delta F$ and the standard diffraction limited antenna resolution of $\Delta x \approx c R / D F_{0}$, where $R, D$, and $F_{0}$ are the range, antenna diameter, and carrier wave frequency. In our system, the antenna diameter ratio and the bandwidth ratio are $(1 \mathrm{~m}) /(25 \mathrm{~m})=0.040$ and $(26 \mathrm{GHz}) /(675 \mathrm{GHz})=$ 0.039 , respectively.

\section{FAST IMAGING}

\subsection{Mirror Motion and Chirp Generation}

There are several potential speed bottlenecks that need to be overcome to achieve fast imaging. The major one in the first-generation $\mathrm{THz}$ imager was the beam scanning speed. In the current system, however, this is no longer the case because of the use of a lightweight fast-nodding flat mirror described in Section 2.1. This mirror is driven by a RGV100BL precision rotary stage from Newport Corp. Although the manufacturer's specifications indicate a maximum acceleration of $1000 \% \mathrm{~s}^{2}$, we have found that this can be exceeded by at least a factor of 15 without significantly affecting the required angular resolution for $\mathrm{THz}$ imaging. For a serpentine scan covering $\pm 3^{\circ}$ in elevation and azimuth (prior to focusing by the main aperture), and with the nodding mirror running at a constant-magnitude angular acceleration $a$, the total imaging time $T$ will be $T=(24 N / a)^{1 / 2}$, where $N$ is the total number of image pixels (assumed to be equal density in $x$ and $y$ ). Typical images contain $N=70 \times 70=4900$, so that for $a=15,000^{\circ} / \mathrm{s}^{2}$, the image time would be $T=2.8$ seconds.

This scan time is not being currently achieved, however, because the speed bottleneck is now the single-pixel dwell time, $t_{d}$, of our FMCW ramp waveform generator. Using a DDS/PLL hybrid synthesizer architecture ${ }^{10}$, with a minimum chirp time of about $0.4 \mathrm{~ms}$ and some overhead for settling and signal processing, the dwell time is $t_{d}=0.5 \mathrm{~ms}$. For a uniform pixel spacing and a constant nodding mirror acceleration magnitude, the dwell time must be shorter than $t_{d} \leq T / 2 N$. Taking $T=2.8 \mathrm{~s}$ and $N=4900$, as above, would require $t_{d}=0.29 \mathrm{~ms}$. We are currently developing a faster chirping synthesizer based on a multiplication of a DDS to exceed this goal. In the meantime, for a dwell time of $0.5 \mathrm{~ms}$, a 4900 pixel image requires about 5 seconds to acquire.

\subsection{Signal Acquisition and Processing}

Other potential frame rate bottlenecks that have not yet been reached are signal acquisition and processing, and the reduction of SNR from ever-shorter chirp times. An experimental analysis of the SNR impact will be done in Section 3. For signal processing, high-speed scanning poses two challenges. One is the ability of software to keep up with the incoming data stream by processing the radar signals, performing image processing, and integrating the threat detection display with a video link. We have found that a fast quad-core processor, running the entire radar imaging routine using LabVIEW software from National Instruments, can readily keep up with real-time imaging demands at the achievable 5 second frame rates.

The second signal processing challenge is that the downconverted IF radar signal frequency $f_{I F}$ increases in proportion to the chirp rate $K$ (in Hz/s) according to $f_{I F}=2 K R / c$. Therefore, care must be taken so that the back-end electronics can accommodate the higher frequency radar signals. A diagram summarizing the detection and processing steps affected by shorter chirp times is shown in Fig. 3. For the THz radar chirp time of $0.44 \mathrm{~ms}$ and bandwidth of $26 \mathrm{GHz}$, the chirp rate is $K=59 \mathrm{MHz} / \mu \mathrm{s}$, and targets at $25 \mathrm{~m}$ standoff yield a frequency around $f_{I F}=10 \mathrm{MHz}$. To simplify and speed up the radar's digital signal processing, an additional downconversion and filtering stage was used to shift the target frequencies within the $2.8 \mathrm{MHz}$ two-sided Nyquist bandwidth of a two-channel (for I and Q) PCI-6132 National Instruments $\mathrm{A} / \mathrm{D}$ converter. At this lower sampling rate, fewer samples per chirp are collected, and decimation by a factor of 3 further restricts the effective final detection bandwidth. With a chirp time of $0.44 \mathrm{~ms}$, the resulting spectral resolution of the signal is in the $\mathrm{kHz}$ range, corresponding to the $0.58 \mathrm{~cm}$ range resolution, and the final spectrum spans a range swath of about $2.3 \mathrm{~m}$. In other words, targets outside a depth of focus spanning a $2.3 \mathrm{~m}$ window at $25 \mathrm{~m}$ standoff will not be detected by the radar. This is a tolerable compromise to make because the Gaussian optics of beam propagation yield a comparable usable range swath: at a distance of $\pm 1.15 \mathrm{~m}$ from the focal point, the beam diameter increases by about $60 \%$. The time-domain signal is compensated for chirp nonlinearity ${ }^{11}$, zero-padded to 512 points, and then processed via fast Fourier transform (FFT) to yield a range-compressed FMCW radar spectrum. Shortening the 
chirp time further to achieve faster frame rates will require either a proportionally higher detection bandwidth, or a reduced depth of focus. The best approach for handling higher chirp speeds is probably increasing the detection bandwidth, since $\mathrm{A} / \mathrm{D}$ converters at $50 \mathrm{MHz}$ or higher, with a large bit depth, are readily available, and any further reduction in the radar depth of focus would compromise the instrument's flexibility.

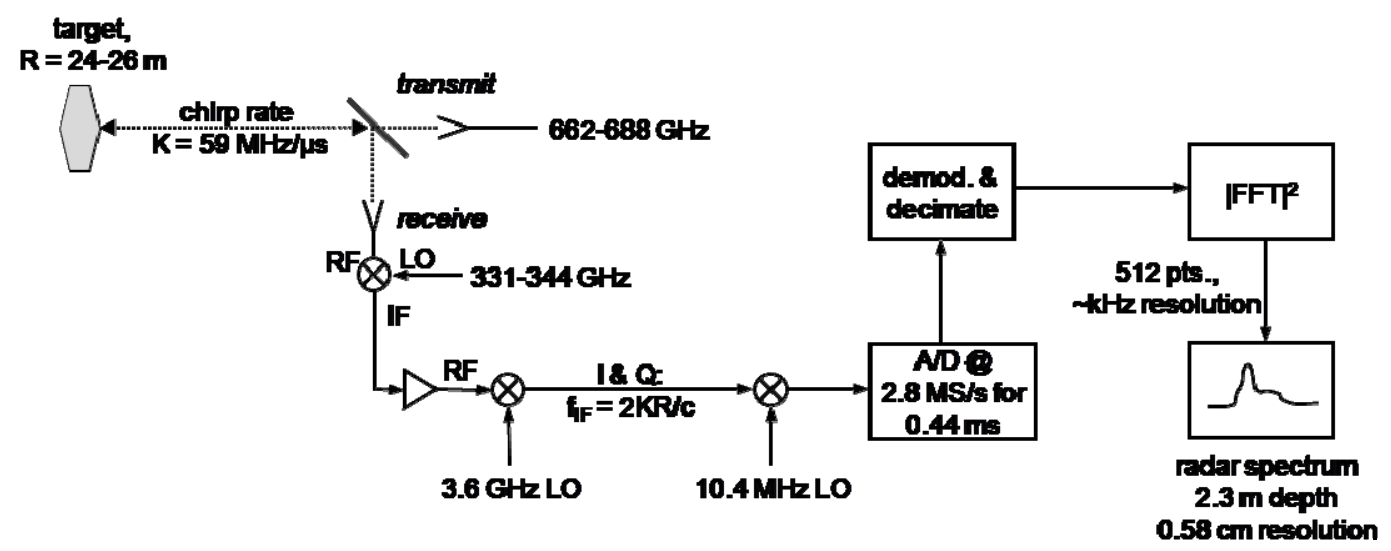

Figure 3: Simplified THz radar signal processing diagram showing key steps that are affected by higher chirp rates. Following the $3.6 \mathrm{GHz}$ downconversion step, the IF frequency is proportional to the chirp rate of $59 \mathrm{MHz} / \mu \mathrm{s}$. Increasing the chirp rate to achieve faster imaging rates will affect the bandwidth and signal processing requirements of the subsequent radar processing steps, which in this case cover a $230 \mathrm{~cm}$ depth of focus with $0.58 \mathrm{~cm}$ resolution.

\subsection{Threat Detection Results and SNR Limits}

The essential signal and image processing steps for generating through-clothes imagery have remained unchanged since the first-generation imager ${ }^{5}$. The procedure involves peak-finding of the radar spectrum obtained for each image pixel, and then reconstructing a "back-surface" topography using the locus of peaks that are at the longest ranges. In other words, the back-surface is the surface that is last encountered by the THz radar beam. This approach is effective because the body, as well as most bulk solid materials that would comprise an explosive device - whether they be metallic or not - are all virtually opaque at the $<0.5 \mathrm{~mW}$ beam powers that the radar employs. (The power density employed is also far below any recognized health hazard threshold.)

Figure 4a provides an example of fast through-clothes threat detection at $25 \mathrm{~m}$ standoff range using the back-surface radar imaging technique. The threat in this case is a mock pipe bomb, consisting of a pair of nail-studded foot-long PVC pipes with an overall diameter of about 1.75 in, as shown in Fig. 4a. The mock explosive device concealed on a torso by a fleece jacket, as well as the $69 \times 65$ pixel back-surface image spanning about $38 \times 38 \mathrm{~cm}$ and acquired in 5 seconds, are also shown in Fig. 4a. The radar chirp time for these data was $0.44 \mathrm{~ms}$, and the fast-rotating mirror's acceleration magnitude was $3,600^{\circ} / \mathrm{s}^{2}$, spanning $\pm 2.5^{\circ}$. Despite operating with somewhat broadened beam profiles, as described in Section 2.2, the THz imaging radar can easily detect the bulge from the concealed pipes. In this way, the THz radar has an ability to perform a standoff pat-down on a person to detect solid objects underneath clothing. 
a)

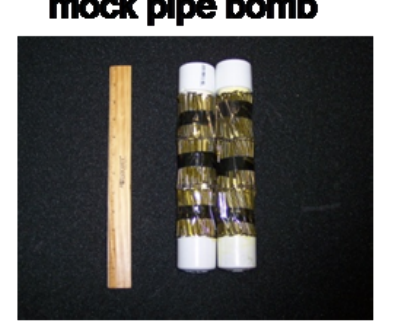

b)

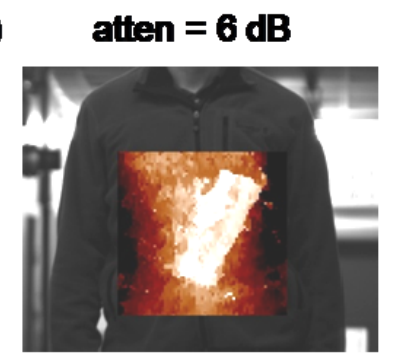

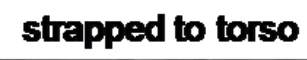

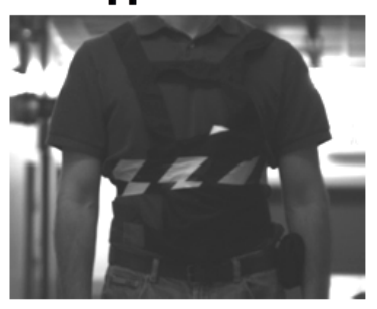

atten $=10 \mathrm{~dB}$

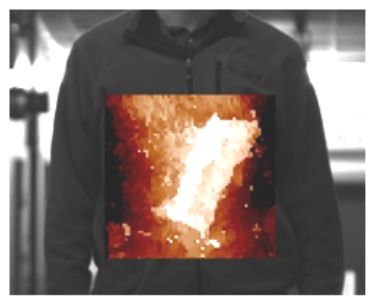

covered by jacket

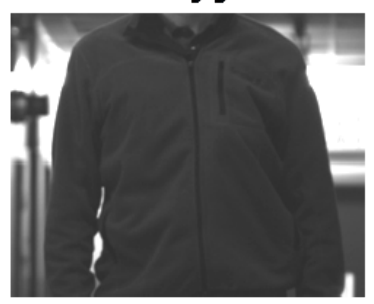

atten $=13 \mathrm{~dB}$

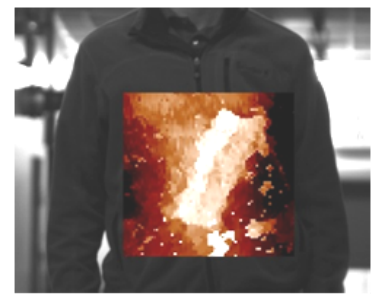

imaged in 5 sec.

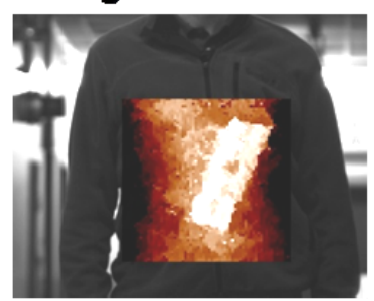

atten $=\mathbf{2 0 ~ d B}$

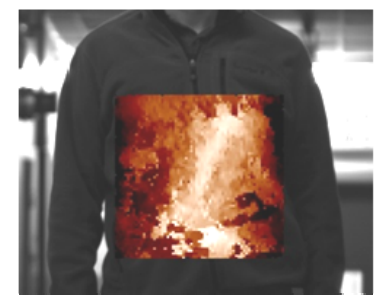

Figure 4: a) Target scenario and back-surface image overlay for through-jacket detection of a mock pipe bomb. The THz radar image was acquired in 5 seconds at a standoff range of $25 \mathrm{~m}$. b) Backsurface image overlays obtained with four levels of signal attenuation to mimic the lower SNR that would result from faster scans. Fair detection quality at $10 \mathrm{~dB}$ attenuation implies that $2 \mathrm{~Hz}$ frame rates will be possible using a single-transceiver $\mathrm{THz}$ radar.

The 5 second imaging time for Fig. 4 a was limited by the minimum attainable chirp time, as explained in Section 3.1, and it is unacceptably long for real-world applications requiring rapid threat discrimination. Not only would a target need to stand in one place during the imaging time so as not to exit the radar's field of view, but motion exceeding about $1 \mathrm{~cm}$ during image capture would cause blurring in the final image. This leads to the question: As chirp times are shortened with better RF circuitry and beam scanning is made faster with more powerful motors or more elegant optical geometries, what are the remaining limits to the imaging speed? One important limit comes from the single-pulse SNR of the radar. Because of the coherent detection technique, SNR of a single-pixel acquisition is proportional to the signal integration time, which is the same as the waveform chirp duration. The individual radar spectra used to generate Fig. 4a exhibit SNRs spanning about 25-45 dB, with the stronger signals primarily occurring in locations where on the angle of incidence between the beam and the target are nearly normal. This implies that the imaging speed can likely be increased by about $15 \mathrm{~dB}$ (to $0.15 \mathrm{sec}$ ) without being SNR-limited, where $10 \mathrm{~dB}$ SNR is taken as the nominal minimum detection threshold.

To investigate this more quantitatively, a series of four additional images from the same scene of Fig. 4a were obtained with additional attenuation placed in the downconverted $(3.6 \mathrm{GHz})$ radar signal path between the submillimeter-wave receiver mixer and the $3.6 \mathrm{GHz}$ low-noise IF amplifier. The attenuations used were $6,10,13$, and $20 \mathrm{~dB}$, and the resulting images are shown in Fig. 4b. Extrapolating the linear relationship between the attenuator values, SNR, and an effective integration time, the images of Fig. $4 \mathrm{~b}$ represent what the radar imagery would look like if the capture time were shortened to be $1.25,0.5,0.25$, and $0.05 \mathrm{~s}$. As this effective imaging time is reduced, the radar's ability to detect and distinguish between the front- and back-surface target layers gradually degrades, with a poor but discernable detection ability at an effective $0.25 \mathrm{sec}$. imaging time ( $13 \mathrm{~dB}$ attenuation), and virtually no through-clothes detection at an effective $50 \mathrm{~ms}$ image time ( $20 \mathrm{~dB}$ attenuation). However, the threat contrast is still quite good at $10 \mathrm{~dB}$ attenuation, corresponding to an effective imaging time of $0.5 \mathrm{~s}$.

Thus we can conclude that the THz radar imager at $25 \mathrm{~m}$ standoff, and with a $1 \mathrm{~m}$ aperture, might be able to attain $2 \mathrm{~Hz}$ frame rates without any change in the front-end $\mathrm{THz}$ electronics. The system improvements needed to achieve this would be a faster-rotating subreflector, a wider bandwidth detector, and a faster microwave chirper. To reach even faster frame rates, either substantial innovation will be required to develop a higher-power, coherent, and compact THz source, and thus increase the SNR, or a means must be developed to simultaneously transmit and receive multiple radar beams on a target. While heterodyne array technology at $\mathrm{THz}$ frequencies is a substantial challenge, the above analysis indicates that only 2-5 transceivers would be required to achieve $\mathrm{THz}$ radar frame rates of $4-10 \mathrm{~Hz}$, or perhaps half that 
number if beam multiplexing approaches are pursued ${ }^{12}$. At these near-video frame rates, the $\mathrm{THz}$ imaging radar may prove valuable in real-world threat detection applications.

\section{CONCLUSIONS}

Early results from JPL's second-generation $\mathrm{THz}$ imaging radar were presented, including effective concealed threat detection in 5 seconds at $25 \mathrm{~m}$ standoff range. This $0.2 \mathrm{~Hz}$ imaging rate is an improvement over the first-generation system by more than an order of magnitude, and its range was extended from 4 to $25 \mathrm{~m}$. This was made possible by a new beam scanning mechanism and optical geometry, a fast microwave chirper, and streamlined signal processing. These subsystems can be further optimized for higher speed and superior beam profile quality. To assess more fundamental limits of the $\mathrm{THz}$ imaging radar speed, measurements with purposefully degraded SNR were also performed. These provided evidence that $>1 \mathrm{~Hz}$ frame rates will be possible with a single $\mathrm{THz}$ transceiver, and there is a potential for near-video imaging rates using only a few-element THz heterodyne array.

\section{AKNOWLEDGEMENTS}

The work was carried out at the Jet Propulsion Laboratory, California Institute of Technology, under a contract with United States Naval Explosive Ordnance Disposal Technology Division. Copyright 2010 California Institute of Technology. Government sponsorship acknowledged.

\section{REFERENCES}

[1] R. Appleby and H.B. Wallace, "Standoff Detection of Weapons and Contraband in the $100 \mathrm{GHz}$ to $1 \mathrm{THz}$ Region," IEEE Trans. on Ant. and Prop. 55, 2944-2956 (2007).

[2] H. B. Liu, Y. Chen, G. J. Bastiaans, and X.-C. Zhang, "Detection and identification of explosive RDX by THz reflection spectroscopy," Opt. Express 14, 415-423 (2006).

[3] For example, see Thruvision Systems Ltd., Brijot Imaging Systems Inc., Sago Systems Inc., and Millivision Corp.

[4] A. Luukanen, P. Helistö, P. Lappalainen, M. Leivo, A. Rautiainen, H. Toivanen, H. Seppä, Z. Taylor, C.R. Dietlein, and E.N. Grossman, "Stand-off passive THz imaging at 8-meter stand-off distance: results from a 64-channel realtime imager," Proc. SPIE 7309, 73090F (2009).

[5] A. Maestrini, J.S. Ward, J.J. Gill, C. Lee, B. Thomas, R.H. Lin, G. Chattopadhyay, and I. Mehdi, "A FrequencyMultiplied Source with more than $1 \mathrm{~mW}$ of Power across the 840-900 GHz Band", to be published IEEE Trans. on Microwave Theory and Techniques (2010).

[6] K. B. Cooper, R. J. Dengler, N. Llombart, T. Bryllert, G. Chattopadhyay, E. Schlecht, J. Gill, C. Lee, A. Skalare, I. Mehdi, P. H. Siegel, "Penetrating 3D Imaging at 4 and 25 Meter Range Using a Submillimeter-Wave Radar", IEEE Trans. Microw. Theory Tech. 56, 2771-2778, (2008).

[7] C. am Weg, W. von Spiegel, R. Henneberger, R. Zimmermann, T. Loeffler, and H.G. Roskos, "Fast Active THz Cameras with Ranging Capabilities," Journal of Infrared, Millimeter, and Terahertz Waves 30(12), 1281-1296 (2009).

[8] D.M. Sheen, T.E. Hall, R.H. Severtsen, D.L. McMakin, B.K. Hatchell, and P.L.J. Valdez, "Active wideband 350GHz imaging system for concealed-weapon detection," Proc. SPIE 7309, 73090I (2009).

[9] N. Llombart, K.B. Cooper, R.J. Dengler, T. Bryllert, and P.H. Siegel, "Confocal Ellipsoidal Reflector System for a Mechanically Scanned Active Terahertz Imager," to be published in IEEE Trans. on Ant. and Prop. (2010).

[10] R. J. Dengler, K. B. Cooper, N. Llombart, G. Chattopadhyay, T. Bryllert, I. Mehdi, and P. H. Siegel, "Toward Realtime Penetrating Imaging Radar at 670 GHz,” 2009 IEEE MTT-S Intl. Microwave Symp. Digest, 941-944 (2009).

[11] R.J. Dengler, K.B. Cooper, G. Chattopadhyay, I. Mehdi, E. Schlecht, A. Skalare, C. Chen, and P.H. Siegel, "600 GHz Imaging Radar with 2 cm Range Resolution,” 2007 IEEE MTT-S Intl. Microwave Symp. Digest, 1371-1374 (2007).

[12] N. Llombart, K.B. Cooper, R.J. Dengler, T. Bryllert, G. Chattopadhyay, and P.H. Siegel, "Time Delay Multiplexing of Two Beams in a THz Imaging Radar,” submitted for publication. 